\title{
2 Emission of Polycyclic Aromatic Hydrocarbons in China
}

SHANSHAN XU, WENXIN LIU, SHU TAO

Laboratory for Earth Surface Processes, College of Environmental Sciences, Peking University, Beijing 100871, China

This supporting information contains detailed information on emission factors used for the estimation (S1), emission of individual PAH species from various sources in China in 2003 (S2), and emission of PAHs from various provinces (S3).

\section{S1 Emission Factors of Major Sources}

The statistics of the EF values used in this study are tabulated in Table S1.

Table S1 Medians (M) and standard errors (SE) of the ln-transformed emission factors of major sources $(\mathrm{mg} / \mathrm{t})$

\begin{tabular}{|c|c|c|c|c|c|c|c|c|c|c|c|c|}
\hline \multirow{3}{*}{$\begin{array}{l}\text { Sources } \\
\\
\text { PAHs }\end{array}$} & \multirow{2}{*}{\multicolumn{2}{|c|}{ Straw }} & \multirow{2}{*}{\multicolumn{2}{|c|}{ Firewood }} & \multirow{2}{*}{\multicolumn{2}{|c|}{$\begin{array}{c}\text { Domestic } \\
\text { Coal }\end{array}$}} & \multirow{2}{*}{\multicolumn{2}{|c|}{$\begin{array}{c}\text { Coking } \\
\text { Coal }\end{array}$}} & \multirow{3}{*}{$\begin{array}{l}\text { Industrial } \\
\text { Coal } \\
\mathrm{M}\end{array}$} & \multirow{3}{*}{$\begin{array}{l}\text { Aluminum } \\
\text { Production } \\
\text { M }\end{array}$} & \multirow{3}{*}{$\begin{array}{l}\text { Transport } \\
\text { Petroleum } \\
\text { M }\end{array}$} & \multirow{3}{*}{$\begin{array}{c}\text { Other } \\
\text { Petroleum } \\
\text { M }\end{array}$} \\
\hline & & & & & & & & & & & & \\
\hline & M & SE & M & SE & M & SE & M & SE & & & & \\
\hline NAP & 9.36 & 0.31 & 9.67 & 0.35 & 9.31 & 0.80 & 8.47 & 0.92 & 3.68 & 8.55 & 8.24 & 6.34 \\
\hline ACY & 5.84 & 0.46 & 8.01 & 0.63 & 8.16 & 1.00 & 6.93 & 0.92 & -2.09 & 1.61 & 1.56 & 1.28 \\
\hline $\mathrm{ACE}$ & 6.75 & 0.36 & 8.17 & 0.47 & 2.49 & 0.20 & 5.49 & 0.39 & -1.30 & 3.49 & -0.78 & 1.31 \\
\hline FLO & 5.89 & 0.44 & 7.82 & 0.53 & 2.62 & 0.21 & 6.45 & 0.70 & -0.79 & 6.67 & 3.26 & 1.87 \\
\hline PHE & 7.83 & 0.31 & 8.44 & 0.26 & 8.01 & 0.70 & 8.34 & 0.59 & 1.80 & 9.37 & 6.59 & 2.06 \\
\hline ANT & 6.03 & 0.37 & 7.35 & 0.35 & 7.35 & 0.63 & 6.89 & 0.66 & -1.58 & 9.08 & 4.60 & 1.84 \\
\hline FLA & 6.96 & 0.30 & 8.02 & 0.21 & 8.37 & 0.71 & 7.27 & 0.29 & 0.94 & 10.02 & 4.81 & 0.57 \\
\hline PYR & 6.93 & 0.30 & 7.85 & 0.21 & 7.84 & 0.64 & 6.78 & 0.35 & -0.36 & 9.84 & 5.02 & -0.42 \\
\hline $\mathrm{BaA}$ & 5.67 & 0.29 & 6.54 & 0.29 & 6.93 & 0.60 & 5.97 & 0.62 & -3.22 & 8.66 & 3.35 & 2.46 \\
\hline CHR & 5.94 & 0.29 & 6.78 & 0.29 & 6.93 & 0.60 & 5.63 & 0.41 & -2.77 & 9.00 & 4.04 & 0.95 \\
\hline $\mathrm{BbF}$ & 5.26 & 0.37 & 6.14 & 0.36 & 7.38 & 0.62 & 5.75 & 0.40 & -1.61 & 8.40 & 2.72 & 0.59 \\
\hline $\mathrm{BkF}$ & 5.66 & 0.31 & 6.19 & 0.25 & 5.66 & 0.49 & 5.06 & 0.29 & -2.35 & 8.25 & 2.50 & 0.56 \\
\hline
\end{tabular}




\begin{tabular}{lcccccccccccc}
\hline BaP & 4.36 & 0.32 & 5.99 & 0.29 & 7.35 & 0.41 & 6.77 & 0.42 & -0.53 & 7.89 & 2.75 & 0.95 \\
IcdP & 4.67 & 0.40 & 6.97 & 0.38 & 7.60 & 0.64 & 6.03 & 0.50 & -2.12 & 6.78 & 2.18 & 1.31 \\
DahA & 2.30 & 0.11 & 5.35 & 0.44 & 8.01 & 0.59 & 4.45 & 0.71 & -2.50 & 6.25 & 0.32 & -1.35 \\
BghiP & 3.96 & 0.47 & 6.91 & 0.27 & 7.13 & 0.58 & 6.03 & 0.44 & -1.90 & 7.15 & 3.07 & 1.59 \\
\hline
\end{tabular}

Note: the numbers of data available for several sources (last four columns) were too small to generate geometric statistics. These sources accounted for less than 5\% of the total PAH16 emission and were not included in the Monte Carlo simulation”

\section{S2 Emissions of Individual PAH Species from Various Sources}

The estimated annual emission rates of $16 \mathrm{PAH}$ species from the major anthropogenic sources in China in 2003 are tabulated in Table S2.

Table S2 Annual emission rates of PAHs from various sources in China (tons)

\begin{tabular}{|c|c|c|c|c|c|c|c|c|c|}
\hline \multirow{2}{*}{ PAHs } & \multirow{2}{*}{$\begin{array}{l}\text { Coking } \\
\text { Industry }\end{array}$} & \multirow{2}{*}{$\begin{array}{c}\text { Domestic } \\
\text { Coal }\end{array}$} & \multirow{2}{*}{$\begin{array}{c}\text { Industrial } \\
\text { Coal }\end{array}$} & \multirow{2}{*}{$\begin{array}{l}\text { Transport } \\
\text { Petroleum }\end{array}$} & \multirow{2}{*}{$\begin{array}{c}\text { Other } \\
\text { Petroleum }\end{array}$} & \multirow{2}{*}{ Straw } & \multicolumn{3}{|c|}{ Aluminum } \\
\hline & & & & & & & Firewood & Production & Total \\
\hline NAP & $1.15 \times 10^{3}$ & $1.45 \times 10^{3}$ & $5.12 \times 10^{1}$ & $3.22 \times 10^{2}$ & $1.14 \times 10^{2}$ & $3.89 \times 10^{3}$ & $3.23 \times 10^{3}$ & $2.88 \times 10^{1}$ & $1.02 \times 10^{4}$ \\
\hline $\mathrm{ACY}$ & $2.47 \times 10^{2}$ & $4.61 \times 10^{2}$ & $1.59 \times 10^{-1}$ & $4.04 \times 10^{-1}$ & $7.27 \times 10^{-1}$ & $1.15 \times 10^{2}$ & $6.12 \times 10^{2}$ & $2.77 \times 10^{-2}$ & $1.44 \times 10^{3}$ \\
\hline ACE & $5.90 \times 10^{1}$ & $1.60 \times 10^{0}$ & $3.52 \times 10^{-1}$ & $3.89 \times 10^{-2}$ & $7.48 \times 10^{-1}$ & $2.86 \times 10^{2}$ & $7.18 \times 10^{2}$ & $1.82 \times 10^{-1}$ & $1.07 \times 10^{3}$ \\
\hline FLO & $1.53 \times 10^{2}$ & $1.82 \times 10^{0}$ & $5.87 \times 10^{-1}$ & $2.23 \times 10^{0}$ & $1.31 \times 10^{0}$ & $1.20 \times 10^{2}$ & $5.10 \times 10^{2}$ & $4.38 \times 10^{0}$ & $7.94 \times 10^{2}$ \\
\hline PHE & $1.01 \times 10^{3}$ & $3.95 \times 10^{2}$ & $7.77 \times 10^{0}$ & $6.18 \times 10^{1}$ & $1.59 \times 10^{0}$ & $8.38 \times 10^{2}$ & $9.42 \times 10^{2}$ & $6.52 \times 10^{1}$ & $3.32 \times 10^{3}$ \\
\hline ANT & $2.37 \times 10^{2}$ & $2.05 \times 10^{2}$ & $2.66 \times 10^{-1}$ & $8.50 \times 10^{0}$ & $1.27 \times 10^{0}$ & $1.39 \times 10^{2}$ & $3.16 \times 10^{2}$ & $4.87 \times 10^{1}$ & $9.56 \times 10^{2}$ \\
\hline FLA & $3.49 \times 10^{2}$ & $5.71 \times 10^{2}$ & $3.29 \times 10^{0}$ & $1.04 \times 10^{1}$ & $3.56 \times 10^{-1}$ & $3.50 \times 10^{2}$ & $6.20 \times 10^{2}$ & $1.24 \times 10^{2}$ & $2.03 \times 10^{3}$ \\
\hline PYR & $2.14 \times 10^{2}$ & $3.33 \times 10^{2}$ & $8.99 \times 10^{-1}$ & $1.29 \times 10^{1}$ & $1.32 \times 10^{-1}$ & $3.42 \times 10^{2}$ & $5.25 \times 10^{2}$ & $1.04 \times 10^{2}$ & $1.53 \times 10^{3}$ \\
\hline $\mathrm{BaA}$ & $9.55 \times 10^{1}$ & $1.34 \times 10^{2}$ & $5.16 \times 10^{-2}$ & $2.44 \times 10^{0}$ & $2.36 \times 10^{0}$ & $9.70 \times 10^{1}$ & $1.41 \times 10^{2}$ & $3.18 \times 10^{1}$ & $5.04 \times 10^{2}$ \\
\hline CHR & $6.74 \times 10^{1}$ & $1.35 \times 10^{2}$ & $8.05 \times 10^{-2}$ & $4.86 \times 10^{0}$ & $5.24 \times 10^{-1}$ & $1.27 \times 10^{2}$ & $1.80 \times 10^{2}$ & $4.51 \times 10^{1}$ & $5.59 \times 10^{2}$ \\
\hline $\mathrm{BbF}$ & $7.59 \times 10^{1}$ & $2.11 \times 10^{2}$ & $2.58 \times 10^{-1}$ & $1.29 \times 10^{0}$ & $3.65 \times 10^{-1}$ & $6.40 \times 10^{1}$ & $9.49 \times 10^{1}$ & $2.48 \times 10^{1}$ & $4.72 \times 10^{2}$ \\
\hline $\mathrm{BkF}$ & $3.82 \times 10^{1}$ & $3.79 \times 10^{1}$ & $1.23 \times 10^{-1}$ & $1.03 \times 10^{0}$ & $3.54 \times 10^{-1}$ & $9.62 \times 10^{1}$ & $1.00 \times 10^{2}$ & $2.13 \times 10^{1}$ & $2.95 \times 10^{2}$ \\
\hline $\mathrm{BaP}$ & $2.11 \times 10^{2}$ & $2.04 \times 10^{2}$ & $7.56 \times 10^{-1}$ & $1.34 \times 10^{0}$ & $5.21 \times 10^{-1}$ & $2.60 \times 10^{1}$ & $8.16 \times 10^{1}$ & $1.48 \times 10^{1}$ & $5.40 \times 10^{2}$ \\
\hline
\end{tabular}


DahA $2.09 \times 10^{1} \quad 3.95 \times 10^{2} \quad 1.06 \times 10^{-1} \quad 1.18 \times 10^{-1} \quad 5.21 \times 10^{-2} \quad 3.33 \times 10^{0} \quad 4.28 \times 10^{1} \quad 2.87 \times 10^{0} \quad 4.66 \times 10^{2}$

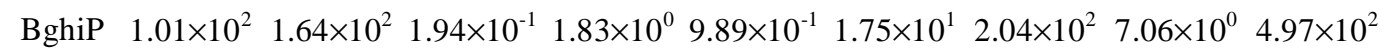

IcdP $\quad 1.01 \times 10^{2} \quad 2.64 \times 10^{2} \quad 1.55 \times 10^{-1} \quad 7.54 \times 10^{-1} \quad 7.51 \times 10^{-1} \quad 3.57 \times 10^{1} \quad 2.17 \times 10^{2} \quad 4.89 \times 10^{0} \quad 6.24 \times 10^{2}$

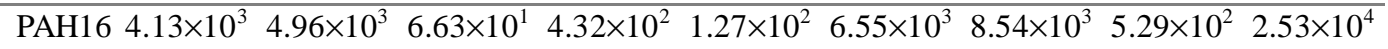

1

\section{S3 Estimated Emissions of PAH16 at Provincial Level}

The emissions of PAH16 from individual provinces, autonomous districts, and municipalities directly under the jurisdiction of the central government in 2003 are tabulated in Table S3.

Table S3 PAH16 emissions at provincial level in China in 2003 (tons)

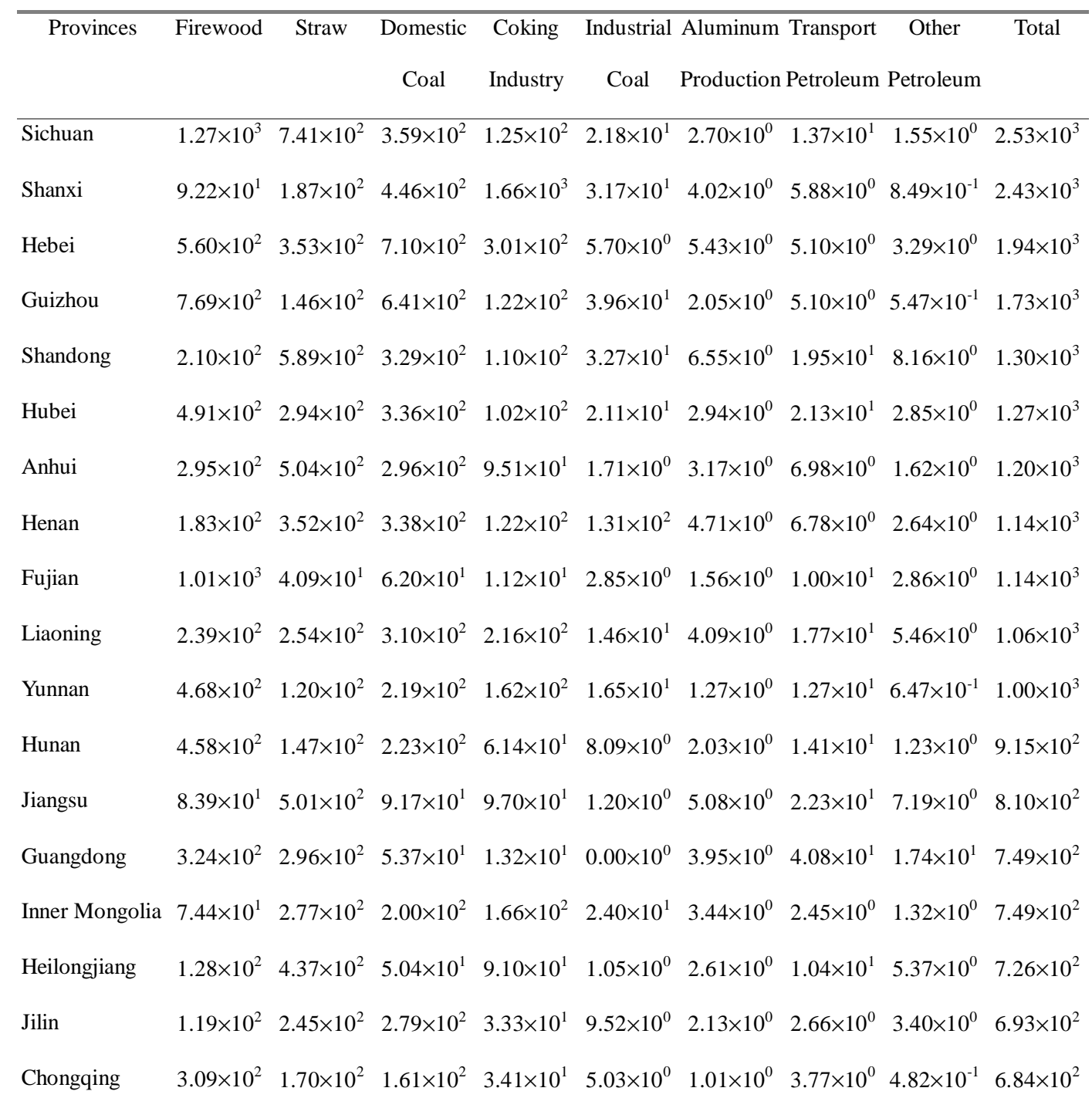




\begin{tabular}{|c|c|c|c|c|c|c|c|c|c|}
\hline Guangxi & $4.40 \times 10^{2}$ & $1.72 \times 10^{2}$ & $2.18 \times 10^{1}$ & $2.29 \times 10^{1}$ & $0.00 \times 10^{0}$ & $1.21 \times 10^{0}$ & $1.11 \times 10^{1}$ & $1.16 \times 10^{0}$ & $6.71 \times 10^{2}$ \\
\hline Shaanxi & $2.28 \times 10^{2}$ & $1.86 \times 10^{2}$ & $1.10 \times 10^{2}$ & $7.21 \times 10^{1}$ & $7.78 \times 10^{0}$ & $1.66 \times 10^{0}$ & $8.44 \times 10^{0}$ & $1.59 \times 10^{0}$ & $6.15 \times 10^{2}$ \\
\hline Jiangxi & $3.16 \times 10^{2}$ & $9.68 \times 10^{1}$ & $1.28 \times 10^{2}$ & $5.31 \times 10^{1}$ & $1.62 \times 10^{0}$ & $1.19 \times 10^{0}$ & $1.36 \times 10^{1}$ & $1.21 \times 10^{0}$ & $6.12 \times 10^{2}$ \\
\hline Xinjiang & $7.57 \times 10^{1}$ & $4.92 \times 10^{1}$ & $3.61 \times 10^{2}$ & $3.14 \times 10^{1}$ & $4.78 \times 10^{0}$ & $1.04 \times 10^{0}$ & $7.33 \times 10^{0}$ & $2.70 \times 10^{0}$ & $5.34 \times 10^{2}$ \\
\hline Gansu & $9.00 \times 10^{1}$ & $1.39 \times 10^{2}$ & $2.12 \times 10^{2}$ & $3.42 \times 10^{1}$ & $4.91 \times 10^{1}$ & $1.25 \times 10^{0}$ & $6.01 \times 10^{0}$ & $8.88 \times 10^{-1}$ & $5.32 \times 10^{2}$ \\
\hline Beijing & $1.83 \times 10^{1}$ & $2.71 \times 10^{1}$ & $2.12 \times 10^{2}$ & $8.61 \times 10^{1}$ & $0.00 \times 10^{0}$ & $8.26 \times 10^{-1}$ & $1.09 \times 10^{1}$ & $3.74 \times 10^{0}$ & $3.59 \times 10^{2}$ \\
\hline Zhejiang & $1.74 \times 10^{2}$ & $5.94 \times 10^{1}$ & $4.34 \times 10^{1}$ & $1.27 \times 10^{1}$ & $7.84 \times 10^{0}$ & $3.30 \times 10^{0}$ & $1.88 \times 10^{1}$ & $6.42 \times 10^{0}$ & $3.26 \times 10^{2}$ \\
\hline Shanghai & $0.00 \times 10^{0}$ & $0.00 \times 10^{0}$ & $4.99 \times 10^{1}$ & $1.87 \times 10^{2}$ & $0.00 \times 10^{0}$ & $1.87 \times 10^{0}$ & $3.06 \times 10^{1}$ & $6.61 \times 10^{0}$ & $2.76 \times 10^{2}$ \\
\hline Hainan & $1.07 \times 10^{2}$ & $5.11 \times 10^{1}$ & $5.11 \times 10^{0}$ & $0.00 \times 10^{0}$ & $2.37 \times 10^{1}$ & $1.66 \times 10^{-1}$ & $4.25 \times 10^{0}$ & $5.18 \times 10^{-1}$ & $1.91 \times 10^{2}$ \\
\hline Tianjin & $2.39 \times 10^{-1}$ & $3.88 \times 10^{1}$ & $7.83 \times 10^{1}$ & $4.43 \times 10^{1}$ & $0.00 \times 10^{0}$ & $1.21 \times 10^{0}$ & $9.70 \times 10^{0}$ & $2.80 \times 10^{0}$ & $1.75 \times 10^{2}$ \\
\hline Taiwan & $0.00 \times 10^{0}$ & $0.00 \times 10^{0}$ & $0.00 \times 10^{0}$ & $1.01 \times 10^{2}$ & $0.00 \times 10^{0}$ & $2.30 \times 10^{0}$ & $5.53 \times 10^{1}$ & $7.17 \times 10^{0}$ & $1.65 \times 10^{2}$ \\
\hline Ningxia & $0.00 \times 10^{0}$ & $4.73 \times 10^{1}$ & $5.08 \times 10^{1}$ & $1.12 \times 10^{1}$ & $2.56 \times 10^{1}$ & $1.37 \times 10^{0}$ & $3.70 \times 10^{0}$ & $7.45 \times 10^{-1}$ & $1.41 \times 10^{2}$ \\
\hline Qinghai & $1.40 \times 10^{1}$ & $2.51 \times 10^{1}$ & $4.39 \times 10^{1}$ & $0.00 \times 10^{0}$ & $3.98 \times 10^{1}$ & $2.81 \times 10^{-1}$ & $5.92 \times 10^{-1}$ & $2.78 \times 10^{-1}$ & $1.24 \times 10^{2}$ \\
\hline Xizang & $2.12 \times 10^{1}$ & $1.47 \times 10^{1}$ & $1.03 \times 10^{1}$ & $0.00 \times 10^{0}$ & $0.00 \times 10^{0}$ & $0.00 \times 10^{0}$ & $8.55 \times 10^{-1}$ & $1.31 \times 10^{-2}$ & $4.70 \times 10^{1}$ \\
\hline Hong Kong & $0.00 \times 10^{0}$ & $0.00 \times 10^{0}$ & $0.00 \times 10^{0}$ & $0.00 \times 10^{0}$ & $0.00 \times 10^{0}$ & $5.49 \times 10^{-1}$ & $1.60 \times 10^{1}$ & $7.00 \times 10^{-1}$ & $1.72 \times 10^{1}$ \\
\hline Macao & $0.00 \times 10^{0}$ & $0.00 \times 10^{0}$ & $0.00 \times 10^{0}$ & $0.00 \times 10^{0}$ & $0.00 \times 10^{0}$ & $0.00 \times 10^{0}$ & $4.43 \times 10^{-1}$ & $2.61 \times 10^{-1}$ & $7.03 \times 10^{-1}$ \\
\hline un & $8.56 \times 10^{3}$ & $6.56 \times 10^{3}$ & $6.43 \times 10^{3}$ & $4.18 \times 10^{3}$ & $5.28 \times 10^{2}$ & $7.70 \times 10^{1}$ & $4.19 \times 10^{2}$ & $1.04 \times 10^{2}$ & $2.69 \times 10^{4}$ \\
\hline
\end{tabular}
tons) exactly with slight differences (c.a. 6\%), because the median values, either national or provincial, were used in the calculation. 